\title{
A Simple Method for Patients Palatal Rugae Duplication in Complete Dentures
}

\author{
${ }^{1}$ Sana Naqvi, ${ }^{2}$ Shankar Pandhrinath Dange, ${ }^{3}$ Smita Arun Khalikar
}

\section{ABSTRACT}

A complete denture should fulfill four basic requirements: esthetics, phonetics, efficiency and comfort. As propagated by Terrell, ${ }^{1}$ dentures should be an aid rather than a hindrance to good speech. Palatal rugae play a vital role in the production of palato lingual sounds. A simple inexpensive method for duplication of patient's palatal rugae is described in this article.

Keywords: Palatal rugae, Heat cure or self cure clear acrylic resin, Condensation silicone putty impression material.

How to cite this article: Naqvi S, Dange SP, Khalikar SA. A Simple Method for Patients Palatal Rugae Duplication in Complete Dentures. Int J Prosthodont Restor Dent 2014;4(2):46-47.

Source of support: Nil

Conflict of interest: None declared

\section{INTRODUCTION}

It is a known fact that three factors play a major role in complete denture are esthetics, mechanics and phonetics. Out of the three mentioned factors, the phonetics is a major factor which is mostly neglected. Neglect to this factor may be attributed to the fact that most of the complete denture patients tend to adjust and return to normal speech after a post insertion practice period of several days to several weeks.

Chierici and Lawson, ${ }^{2}$ Palmer, ${ }^{3}$ and Troffer and Beder ${ }^{4}$ have described the principles behind speech problems. To understand how denture changes improve speech function one should know the concept of air turbulence. A method for production of air turbulence is duplication of patient's rugae pattern in the complete denture. Palatal rugae play a important role in production of palato lingual sound. A simple technique is described in this article, to duplicate palatal rugae pattern that can be customized for each patient using condensation silicone putty impression material.

\footnotetext{
${ }^{1}$ Postgraduate Student, ${ }^{2}$ Professor and Head, ${ }^{3}$ Associate Professor

${ }^{1-3}$ Department of Prosthodontics, Government Dental College and Hospital, Aurangabad, Maharashtra, India
}

Corresponding Author: Sana Naqvi, Postgraduate Student Department of Prosthodontics, Government Dental College and Hospital, Aurangabad, Maharashtra, India, Phone: 07122590097 e-mail: sananaqui222@yahoo.com

\section{PROCEDURE}

Mark rugae patterns on maxillary final cast using permanent marker (Fig. 1).

Apply separating medium on the cast. Fabricate a clear acrylic (DPI cold Cure, Translucent, Dental Products of India) record base on the cast. The markings are seen through the transparent resin base plate on the cast (Fig. 2). The thickness of the record base should not exceed $2 \mathrm{~mm}$.

Observe the pattern through the record base and using a straight fissure bur inscribe the rugae pattern on the record base to create roughness that will retain the putty impression material (Fig. 3).

Make the tentative jaw relation and do the teeth arrangement. Do the trial denture verification.

Demount the cast from articulator.

Mix the putty with the catalyst (Zetaplus, Zermack Clinical C-Silicone impression material, Italy) and make small threads out of the kneaded material and adapt according to the patient's rugae pattern on the record base (Fig. 4).

The diameter of the threads can vary according to the variations in the thickness of rugae.

Do the denture fabrication in conventional manner. Do the flasking and counterflasking. In the counterflask, the negative imprint of the rugae is duplicated.

Following this do the dewaxing and remove the clear acrylic base plate along with the putty threads and do the packing with heat cure denture base material (Acralyn-'H', Pink, Asian Acrylates, India) and do the curing. The rugae pattern is duplicated in the denture (Fig. 5).

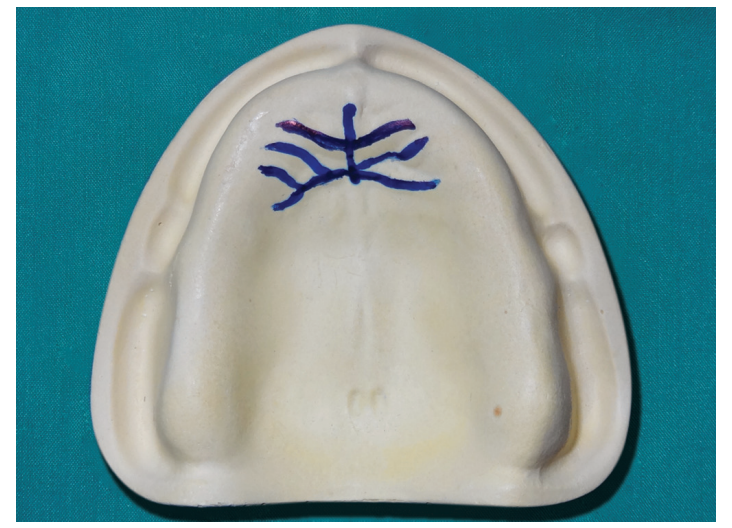

Fig. 1: Palatal rugal marked on cast 


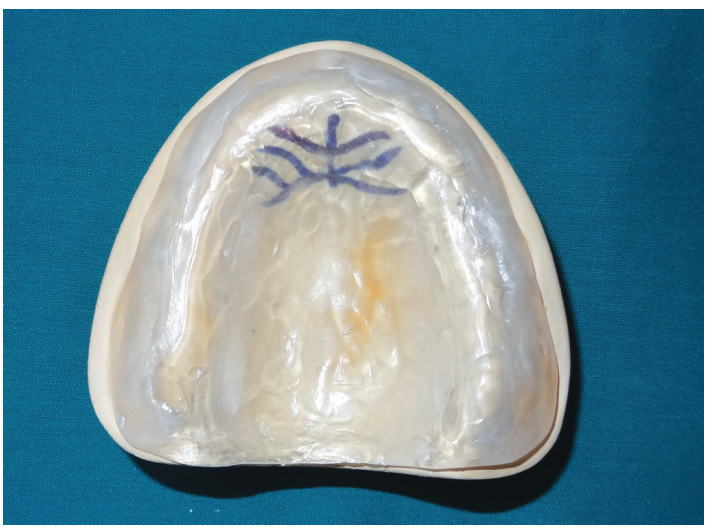

Fig. 2: Clear acrylic record base

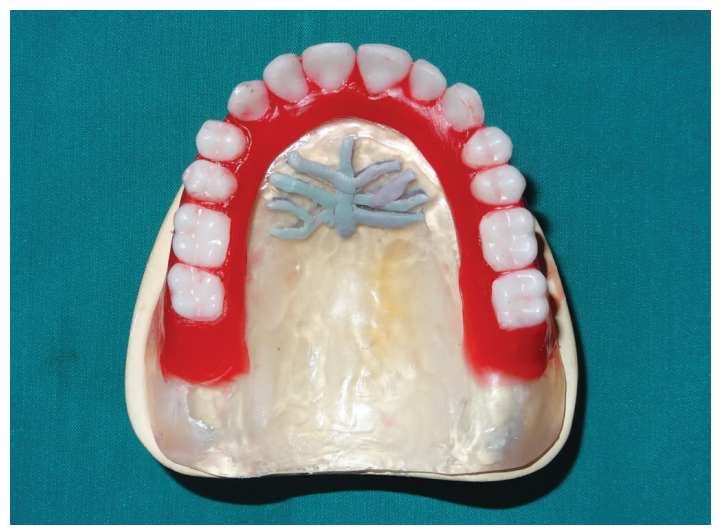

Fig. 4: Condensation silicone used to duplicate rugae pattern

\section{DISCUSSION}

In the literature, many methods of improving speech have been suggested and evaluated. Snow ${ }^{5}$ suggests thickening and contouring the area lingual to the collars of maxillary incisors for speech facilitation. Pound ${ }^{6}$ advocated that the entire lingual aspect of maxillary denture should be contoured to simulate the normal palate, if proper phonetics is to be attained. Many authors suggest palatal rugae fabrication to facilitate speech.

There are many methods of carving palatal rugae on the complete dentures. This can be done using plastic palate forms, corrugated metal palate and free hand carving of anatomic palate. The procedure of electroplating to form metal palate that duplicates patient's palate, is limited, in that it does not apply impression of maxillary cast to make custom acrylic resin pattern to capture patient's anatomy. But this involves making additional impression or duplication of cast. Missing lingual contours of denture teeth should be added during wax up of trial dentures in this method. Use of palatogram has also been done to modify palatal portion of denture. The procedure described here involves use of self cure clear acrylic resin and condensation silicone putty

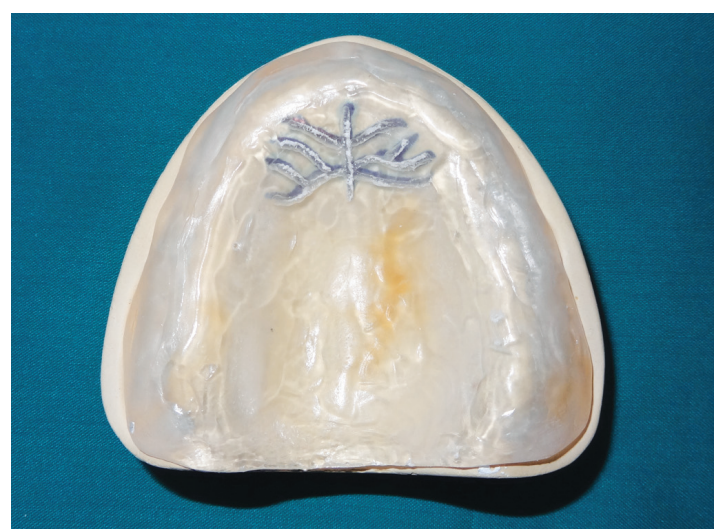

Fig. 3: Palatal rugae inscribed on record base using straight fissure bur

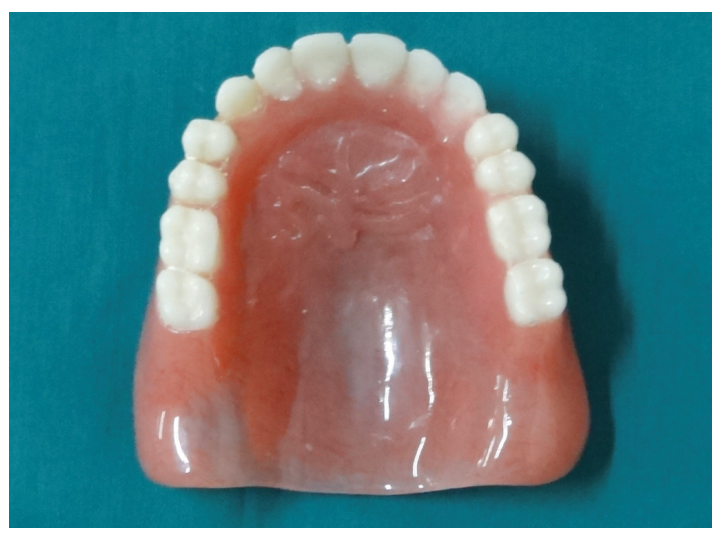

Fig. 5: Processed denture with rugae duplicated

impression material, which easily duplicates the width and thickness of rugae to an acceptable extent. The amount of putty used is very less so it is cost-effective. Putty can be molded and shaped easily to customize individual rugae. Once set it is hard enough to withstand the processing procedure without undergoing distortion in contrast to other materials, such as waxes. As the rugae pattern is inscribed on the base, plate with the bur there is mechanical retention of putty and negligible chances of displacement. The rugae duplicated in finished denture does not interfere with speech.

\section{REFERENCES}

1. Terrell WH. Fundamentals important to good complete denture construction. J Prosthet Dent 1958;8:740-752.

2. Palmer JM. Analysis of speech in prosthodontic practice. J Prosthet Dent 1974 Jun;31(6):605-614.

3. Chierici G, Lawson L. Clinical speech considerations in prosthodontics: perspectives of the prosthodontist and speech pathologist. J Prosthet Dent 1973 Jan;(1):29-39.

4. Troffer C, Beder OE. Immediate dentures and speech deficiencies. Dent Prog 1961;1:264.

5. Snow GB. The faces of proper conformation of the lingual surfaces of dental plates. Advertiser (Dental) XX 1899:51-54.

6. Pound E. Aesthetics and phonetics in full denture construction. Dent J Aust 1951 Mar;23(3):126-134. 